November 14, 2018

\title{
Theta Functions Associated with the Affine Root Systems and the Elliptic Ruijsenaars Operators
}

\author{
Yasushi Komori 冈 \\ Department of Physics, Graduate School of Science, \\ University of Tokyo, \\ Hongo 7-3-1, Bunkyo, Tokyo 113, Japan. \\ (Received: $\quad$ )
}

\begin{abstract}
We study a family of mutually commutative difference operators associated with the affine root systems. These operators act on the space of meromorphic functions on the Cartan subalgebra of the affine Lie algebra. We show that the space spanned by the characters of a fixed positive level is invariant under the action of these operators.
\end{abstract}

\footnotetext{
*E-mail: komori@monet.phys.s.u-tokyo.ac.jp
} 


\section{Introduction}

In [27], a family of mutually commutative operators, whose coefficients consist of theta functions, were introduced as a relativistic quantum many-body system, i.e., an elliptic difference analogue of the Calogero-Sutherland model. Since then, these operators have been studied extensively from various points of view, especially from the analogy with the Macdonald operators. The eigenvectors of the Macdonald operators are a two-parameter extension of the Schur functions or the characters of finite dimensional simple Lie algebras. Then it is natural to expect this structure in the elliptic case. In fact, it was clarified in 11, 12 that the elliptic analogues of type $A_{l}^{(1)}$ and $C_{2}^{(1)}$ have an invariant subspace in the meromorphic functions and that this space is actually spanned by the characters of the corresponding affine Lie algebra. These facts are found through the studies of the intertwining vectors between the face models and the vertex models. Independently, in [9], the Boltzmann weight of the matrix elements of Belavin's elliptic $R$-matrix was calculated making use of this fact implicitly.

In a series of Cherednik's papers [3, 6, 7], it has been proved out that the double affine Hecke algebra plays an essential role in the Macdonald theory. There are some algebras that are considered to describe the structure of the elliptic analogues [5, 8, 10, 31]. In this paper, we employ yet another approach or the root algebra to these operators. Following the previous work 21] where we studied nontwisted cases, we construct a family of mutu-

ally commuting difference operators associated with arbitrary affine root systems. These operators are shown to act on the vector space of the Weyl group invariant meromorphic functions and, furthermore, on the space spanned by the characters of a fixed positive level.

This paper is organized as follows: In section 2, we prepare the notations and definitions used in this paper. In section 3, we define the root algebras that was introduced by Cherednik in the development of the theory of the affine Hecke algebras. In section 4, we demonstrate some examples of the generators of a commutative subalgebra in the root algebras. In section 5, we give some representations of the root algebras with a spectral parameter, which consist of Jacobi's theta functions and act on the meromorphic 
functions on the Cartan subalgebra. We show that when we assign a special value to the spectral parameter, the difference operators preserve the Weyl group invariant subspace. By construction, they form a commutative family. In section 6, we calculate the explicit forms of these operators at this spectral parameter and observe that they can be regarded as an elliptic analogue of the Macdonald operators. In twisted cases, we have the difference and quantum version of the systems that is recently proposed and is dealt in terms of the Lax formalism [1]. We also prove that the generators are algebraically independent and thus the commutative subalgebra is isomorphic to a polynomial ring. In section 7 , we show the main theorem (Theorem 7.5) that they have an infinite dimensional invariant subspace (finite rank submodule) of the theta functions of positive level, where the key of the proof is due to [9,19. The last section is devoted to the concluding remarks.

To end this section, we present two elliptic difference operators which take the simplest form among the generators respectively in the root systems of type $A_{l-1}^{(1)}$ and $A_{2 l}^{(2)}$ :

$$
\begin{aligned}
\hat{Y}_{A_{l-1}^{(1)}}^{-\lambda_{1}} & =\sum_{j=1}^{l} \prod_{j \neq k}^{l} \frac{\vartheta_{1}\left(x_{j}-x_{k}-\mu\right)}{\vartheta_{1}\left(x_{j}-x_{k}\right)} t_{j}(\kappa) \prod_{j=1}^{l} t_{j}(-\kappa / l) \\
\hat{Y}_{A_{2 l}^{(2)}}^{-\lambda_{1}} & =\sum_{j=1}^{l}\left(\prod_{\substack{k=1 \\
k \neq j}}^{l} \frac{\vartheta_{1}\left(x_{j}-x_{k}-\mu\right)}{\vartheta_{1}\left(x_{j}-x_{k}\right)} \frac{\vartheta_{1}\left(x_{j}+x_{k}-\mu\right)}{\vartheta_{1}\left(x_{j}+x_{k}\right)}\right)\left(\prod_{r=0}^{3} \frac{\vartheta_{r+1}\left(x_{j}-\nu_{r}\right)}{\vartheta_{r+1}\left(x_{j}\right)} \frac{\vartheta_{r+1}\left(x_{j}+\kappa / 2-\bar{\nu}_{r}\right)}{\vartheta_{r+1}\left(x_{j}+\kappa / 2\right)}\right) t_{j}(\kappa) \\
+\sum_{j=1}^{l} & \left(\prod_{\substack{k=1 \\
k \neq j}}^{l} \frac{\vartheta_{1}\left(-x_{j}-x_{k}-\mu\right)}{\vartheta_{1}\left(-x_{j}-x_{k}\right)} \frac{\vartheta_{1}\left(-x_{j}+x_{k}-\mu\right)}{\vartheta_{1}\left(-x_{j}+x_{k}\right)}\right)\left(\prod_{r=0}^{3} \frac{\vartheta_{r+1}\left(-x_{j}-\nu_{r}\right)}{\vartheta_{r+1}\left(-x_{j}\right)} \frac{\vartheta_{r+1}\left(-x_{j}+\kappa / 2-\bar{\nu}_{r}\right)}{\vartheta_{r+1}\left(-x_{j}+\kappa / 2\right)}\right) t_{j}(-\kappa) \\
& \left.-\sum_{p=0}^{3}\left(\frac{\pi}{\vartheta_{1}^{\prime}(0)}\right)^{2} \frac{\prod^{3}(-\mu) \vartheta_{1}(-\kappa-\mu)}{\vartheta_{r=0}(-\kappa)} \vartheta_{r+1}\left(-\kappa-\nu_{\pi_{p} r}\right) \vartheta_{r+1}\left(-\bar{\nu}_{\pi_{p} r}\right)\right) \\
& \quad\left(\prod_{j=1}^{N} \frac{\vartheta_{p+1}\left(x_{j}-\kappa / 2-\mu\right)}{\vartheta_{p+1}\left(x_{j}-\kappa / 2\right)} \frac{\vartheta_{p+1}\left(-x_{j}-\kappa / 2-\mu\right)}{\vartheta_{p+1}\left(-x_{j}-\kappa / 2\right)}\right)
\end{aligned}
$$

Here we have realized the root systems in $\mathbb{C}^{l}$ in the standard way, $\vartheta_{j}(x)=\vartheta_{j}(x ; \tau)$ is the Jacobi theta function and $t_{i}(\kappa)$ is a translation of the variable $x_{i}$ by $\kappa . \pi_{r}(r=0,1,2,3)$ denotes the permutation: $\pi_{0}=i d, \pi_{1}=(01)(23), \pi_{2}=(02)(13)$, and $\pi_{3}=(03)(12)$. The parameters $\kappa, \mu, \nu_{r}$ and $\bar{\nu}_{r}(r=0,1,2,3)$ are arbitrary constants. The operator (1.1) was introduced in [27] together with the whole family of commuting difference operators, while 
the operator (1.2) was conjectured to be a member of a commutative family in [32, 33]. The $A_{2 l}^{(2)}$-type model was referred to as $D$-type or $B C$-type in previous papers.

If we set $\kappa=l \mu / k$ in $A_{l-1}^{(1)}$ case and $\kappa=(\nu+2 \bar{\nu}+2(l-1) \mu) / k$ in $A_{2 l}^{(2)}$ case, where $\nu=\sum \nu_{r}$ and $\bar{\nu}=\left(\sum \bar{\nu}_{r}\right) / 2$, then these operators have an invariant subspace which consists of the characters of level $k$ corresponding to each affine Lie algebra. When the parameters $\mu, \nu, \bar{\nu}$ are set to be unity, we see that $\kappa$ reduces to $h^{\vee} / k$ where $h^{\vee}$ is the dual Coxeter number. For the derivation of these facts in each case, see [11, 13, 14, 20, 22].

\section{Affine Root Systems}

We give some well-known facts about the affine root systems and the affine Weyl groups [2, 4,15, which are the standard tools in the theory of the affine Hecke algebras. Some of the definitions are slightly changed and extended so that they include the twisted affine root systems. Most of the notations are due to [16.

Let $\mathfrak{g}=\mathfrak{g}(A)$ be the affine Lie algebra associated with the generalized Cartan matrix $A$ of type $X_{N}^{(r)}, \mathfrak{h}$ its Cartan subalgebra, $\operatorname{dim} \mathfrak{h}=l+1$ the rank of $\mathfrak{g}, I=\{0, \ldots, l\}$ a set of indices, $\Pi=\left\{\alpha_{i} \mid i \in I\right\} \subset \mathfrak{h}^{*}$ the set of simple roots, $\Pi^{\vee}=\left\{\alpha_{i}^{\vee} \mid i \in I\right\} \subset \mathfrak{h}$ the set of simple coroots, $\Delta$ the root system, $Q$ and $Q^{\vee}$ the root and coroot lattices, $P$ and $P^{\vee}$ the weight and coweight lattices:

$$
\begin{aligned}
& Q=\bigoplus_{i \in I} \mathbb{Z} \alpha_{i} \subset P=\bigoplus_{i \in I} \mathbb{Z} \Lambda_{i} \oplus \mathbb{C} \delta \subset \mathfrak{h}^{*} ; \\
& Q^{\vee}=\bigoplus_{i \in I} \mathbb{Z} \alpha_{i}^{\vee} \subset P^{\vee}=\bigoplus_{i \in I} \mathbb{Z} \Lambda_{i}^{\vee} \oplus \mathbb{C} K \subset \mathfrak{h},
\end{aligned}
$$

where $\left\langle\alpha_{i}, \Lambda_{j}^{\vee}\right\rangle=\delta_{i j},\left\langle\Lambda_{i}, \alpha_{j}^{\vee}\right\rangle=\delta_{i j}, d=\Lambda_{0}^{\vee}$. Since the normalized invariant form is nondegenerate on $\mathfrak{h}$, we have an isomorphism $\nu: \mathfrak{h} \rightarrow \mathfrak{h}^{*}$ defined by

$$
\left\langle\nu(h), h_{1}\right\rangle=\left(h \mid h_{1}\right), \quad h, h_{1} \in \mathfrak{h},
$$

and the induced bilinear form (.|.) on $\mathfrak{h}^{*}$. Let $\stackrel{\circ}{I}=\{1, \ldots, l\}, \stackrel{\circ}{\Pi}=\left\{\alpha_{i} \mid i \in \stackrel{\circ}{I}\right\}$ and $\stackrel{\circ}{\Pi} \vee=$ $\left\{\alpha_{i}^{\vee} \mid i \in \stackrel{\circ}{I}\right\}$. Let $\stackrel{\circ}{\mathfrak{h}}^{*}$ be the subspace of $\mathfrak{h}^{*}$ spanned by $\stackrel{\circ}{\Pi}$ over $\mathbb{C}$. For $\lambda \in \mathfrak{h}^{*}$, denote by $\bar{\lambda}$ 
the orthogonal projection of $\lambda$ on $\stackrel{\circ}{h}^{*}$. Let $\stackrel{\circ}{Q}$ be the sublattice of $Q$ generated by $\stackrel{\circ}{\Pi}$ and $\stackrel{\circ}{P}$ the projection of $P$ on $\stackrel{\circ}{h}^{*}$. The dual notions $\stackrel{\circ}{\mathfrak{h}}, \bar{h}, \stackrel{\circ}{Q}^{\vee}$ and $\stackrel{\circ}{P}^{\vee}$ are defined similarly:

$$
\begin{aligned}
& \stackrel{\circ}{Q}=\bigoplus_{\substack{i \in I \\
I}} \mathbb{Z} \alpha_{i} \subset \stackrel{\circ}{P}=\bigoplus_{\substack{i \in I \\
i \in I}} \mathbb{Z} \overline{\Lambda_{i}} \subset \stackrel{\circ}{\mathfrak{h}}^{*} ; \\
& \stackrel{\circ}{Q} \vee
\end{aligned}
$$

For $\alpha \in \Delta^{r e}$, let $r_{\alpha}$ be a reflection defined by

$$
r_{\alpha}(\lambda):=\lambda-\left\langle\lambda, \alpha^{\vee}\right\rangle \alpha, \quad \lambda \in \mathfrak{h}^{*}
$$

The Weyl group $\stackrel{\circ}{W}$ is generated by the fundamental reflections $\left\{r_{i}:=r_{\alpha_{i}} \mid i \in \stackrel{\circ}{I}\right\}$ on $\mathfrak{h}^{*}$ and the affine Weyl group $W$ is generated by $\left\{r_{i} \mid i \in I\right\}$. The defining relations are given by $r_{i}^{2}=i d$ and the Coxeter relations:

$$
\left(r_{i} r_{j}\right)^{m_{i j}}=i d, \quad \text { for } i \neq j \in I,
$$

where $m_{i j}=2$ if $\alpha_{i}$ and $\alpha_{j}$ are disconnected in the Dynkin diagram $S(A)$ and $m_{i j}=3,4,6$ if $1,2,3$ lines respectively connect $\alpha_{i}$ and $\alpha_{j}$ in $S(A)$. We note that there is no Coxeter relation in the affine root systems of rank 2. For $\alpha \in \stackrel{\circ}{\mathfrak{h}}^{*}$, we define endomorphisms $t_{\alpha}, t_{\alpha}^{\iota}$ of the vector space $\mathfrak{h}^{*}$ for $\kappa \in \mathbb{C}$ by (cf. [5])

$$
\begin{aligned}
t_{\alpha}(\lambda) & :=\lambda+\langle\lambda, K\rangle \alpha-\left((\lambda \mid \alpha)+\frac{1}{2}|\alpha|^{2}\langle\lambda, K\rangle\right) \delta \\
t_{\alpha}^{\iota}(\lambda) & :=\lambda-\kappa(\lambda \mid \alpha) \delta .
\end{aligned}
$$

Here $t_{\alpha}^{\iota}$ is associated with an endomorphism of $\mathfrak{h}^{*}, \iota(\lambda):=\lambda+(\kappa-1)\langle\lambda, d\rangle \delta$ as follows:

$$
\iota \circ t_{\alpha}(\stackrel{\circ}{\lambda}+m \delta)=t_{\alpha}^{\iota}(\stackrel{\circ}{\lambda}+m \delta)
$$

Let $a_{i}$ and $a_{i}^{\vee}$ be the labels of the Dynkin diagram from Table Aff in [16]. Note that $a_{0}=2$ if $A$ is of type $A_{2 l}^{(2)}$ and $a_{0}=1$ otherwise. Let $\theta:=\delta-a_{0} \alpha_{0} \in \stackrel{\circ}{\Delta}{ }_{+}, M:=\nu\left(\mathbb{Z}\left(\stackrel{\circ}{W} \cdot \theta^{\vee}\right)\right) \subset \stackrel{\circ}{\mathfrak{h}}$, $T_{M}$ the corresponding group of translations of $M$. Then

Proposition 2.1. The group $W$ is the semidirect product $W=\stackrel{\circ}{W} \ltimes T_{M}$. 
For $\alpha \in \Delta^{r e}$, let $\gamma_{\alpha}:=r$ if $\alpha \in \Delta_{l}$ and $\gamma_{\alpha}:=1$ otherwise. Then the real roots are written as

$$
\Delta^{r e}= \begin{cases}\left\{\alpha+n \gamma_{\alpha} \delta \mid \alpha \in \stackrel{\circ}{\Delta}, n \in \mathbb{Z}\right\}, & \text { if } A \text { is not of type } A_{2 l}^{(2)} ; \\ \left\{\alpha+n \gamma_{\alpha} \delta \mid \alpha \in \stackrel{\circ}{\Delta}, n \in \mathbb{Z}\right\} \cup & \\ \left\{\frac{1}{2}(\alpha+(2 n-1) \delta) \mid \alpha \in \stackrel{\circ}{\Delta}_{l}, n \in \mathbb{Z}\right\}, & \text { if } A \text { is of type } A_{2 l}^{(2)} .\end{cases}
$$

Let $\widehat{M}:=\left\{\lambda \in \stackrel{\circ}{\mathfrak{h}}^{*} \mid \alpha \in \Delta^{r e},(\alpha \mid \lambda) \in \gamma_{\alpha} \mathbb{Z}\right\}$. Then we see that $\widehat{M} \subset \stackrel{\circ}{P}$ and $T_{\widehat{M}}$ is normalized by $\stackrel{\circ}{W}$.

Definition 2.2. The extended affine Weyl group $\widehat{W}$ is the semidirect product $\widehat{W}:=\stackrel{\circ}{W} \ltimes$ $T_{\widehat{M}}$.

The lattice $\widehat{M}$ is defined so that the extended affine Weyl group acts on $\Delta$. Here are the explicit description of $\widehat{M}$ and its canonical basis $\left\{\lambda_{i} \mid i \in \stackrel{\circ}{I}\right\}$ :

$$
\widehat{M}=\left\{\begin{array}{ll}
\nu\left(\stackrel{\circ}{P^{\vee}}\right), & \text { if } r=1 ; \\
\stackrel{\circ}{P}, & \text { otherwise, }
\end{array} \quad \lambda_{i}= \begin{cases}\nu\left(\overline{\Lambda_{i}^{\vee}}\right), & \text { if } r=1 \\
\overline{\Lambda_{i}}, & \text { otherwise }\end{cases}\right.
$$

We also use $\widehat{M}_{-}:=\oplus_{i \in I} \circ \mathbb{Z}_{\leq 0} \lambda_{i}$. The action of $\widehat{W}$ is naturally induced on $\mathfrak{h}$ via the form $\langle\cdot, \cdot\rangle$.

Let $\Omega$ be the subgroup of $\widehat{W}$ which stabilizes the affine Weyl chamber $C$.

Proposition 2.3. The subgroup $\Omega$ is isomorphic to $\widehat{W} / W \simeq T_{\widehat{M}} / T_{M}$ thus Abelian. The extended affine Weyl group $\widehat{W}$ is isomorphic to the semi-direct product $W \rtimes \Omega$.

Definition 2.4. 1. The length $\ell(w)$ of $w \in W$ is the length $\ell$ of the reduced decomposition:

$$
\begin{aligned}
& w=r_{i_{1}} \ldots r_{i_{\ell}}, \quad \text { for } \quad i_{k} \in I, \\
& \ell(i d)=0 .
\end{aligned}
$$


2. The length $\ell(\hat{w})$ of $\hat{w} \in \widehat{W}$ is the number of the positive roots made negative by $\hat{w}^{-1}$ :

$$
\begin{aligned}
& \ell(\hat{w}):=\left|\Delta_{\hat{w}}\right|, \\
& \Delta_{\hat{w}}:=\left\{\alpha \in \Delta_{+} \cap-\hat{w} \Delta_{+}\right\},
\end{aligned}
$$

which is equivalent to the definition $\ell(w)$ for $w \in W$. The reduced decomposition of $\hat{w} \in \widehat{W}$ is $\hat{w}=w \omega=r_{i_{1}} \ldots r_{i_{\ell}} \omega$, where $\omega \in \Omega$ and $\ell=\ell(\hat{w})=\ell(w)$.

The set $\Delta_{\hat{w}}$ is explicitly described as $\Delta_{\hat{w}}=\left\{\alpha^{1}=\alpha_{i_{1}}, \alpha^{2}=r_{i_{1}}\left(\alpha_{i_{2}}\right), \ldots, \alpha^{\ell}=\right.$ $\left.w r_{i_{\ell}}\left(\alpha_{i_{\ell}}\right)\right\}$. By definition, $\Delta_{\hat{w}}$ is independent of reduced expressions. One sees that $\Omega=\{\omega \in \widehat{W}, \ell(\omega)=0\}$.

Definition 2.5. A weight $\lambda \in \widehat{M}$ is said to be minuscule if $\Delta_{t_{-\lambda}} \subset \stackrel{\circ}{\Delta}_{+}$.

We use the following useful formulas, which can be easily derived from the definitions above:

$$
\begin{aligned}
& \Delta_{t_{\lambda_{-}}}=\left\{\begin{array}{l}
\left\{\alpha-n \gamma_{\alpha} \delta \mid \alpha \in \stackrel{\circ}{\Delta}_{+}, 0 \geq n>\frac{1}{\gamma_{\alpha}}\left(\lambda_{-} \mid \alpha\right)\right\}, \\
\left\{\alpha-n \gamma_{\alpha} \delta \mid \alpha \in \stackrel{\circ}{\Delta}_{+}, 0 \geq n>\frac{1}{\gamma_{\alpha}}\left(\lambda_{-} \mid \alpha\right)\right\} \cup \\
\quad\left\{\frac{1}{2}(\alpha-(2 n-1) \delta) \mid \alpha \in\left({\left.\stackrel{\circ}{\Delta_{+}}\right)}_{l}, 0 \geq n>\frac{1}{2}\left(\lambda_{-} \mid \alpha\right)\right\},\right.
\end{array}\right. \\
& \ell\left(t_{\lambda_{-}}\right)= \begin{cases}\sum_{\alpha \in \AA_{+}}\left|\frac{1}{\gamma_{\alpha}}\left(\alpha \mid \lambda_{-}\right)\right|, & \text {if } A \text { is not of type } A_{2 l}^{(2)} ; \\
\sum_{\alpha \in \AA_{+}}\left|\left(\alpha \mid \lambda_{-}\right)\right|, & \text {if } A \text { is of type } A_{2 l}^{(2)},\end{cases} \\
& \ell\left(r_{j} t_{-\lambda_{i}}\right)=\ell\left(t_{-\lambda_{i}}\right)+1 \text {, } \\
& \ell\left(r_{i} t_{-\lambda_{i}}\right)=\ell\left(t_{-\lambda_{i}}\right)-1, \\
& \ell\left(t_{\lambda_{-}} w\right)=\ell\left(t_{\lambda_{-}}\right)+\ell(w), \\
& \ell\left(t_{\lambda_{-}+\lambda_{-}^{\prime}}\right)=\ell\left(t_{\lambda_{-}}\right)+\ell\left(t_{\lambda_{-}^{\prime}}\right),
\end{aligned}
$$

if $A$ is not of type $A_{2 l}^{(2)}$;

if $A$ is of type $A_{2 l}^{(2)}$,

where $i \neq j \in \stackrel{\circ}{I}, \lambda_{-}, \lambda_{-}^{\prime} \in \widehat{M}_{-}, w \in \stackrel{\circ}{W}$. 


\section{Root Algebras}

We shall define the root algebras after Cherednik [4]. Let $\mathcal{T}$ be the tensor algebra over $\mathbb{C}$ generated by independent variables $\left\{R_{\alpha} \mid \alpha \in \Delta^{r e}\right\}$. Then the action of $\hat{w} \in \widehat{W}$ on $\Delta^{r e}$ induces an action on $\mathcal{T}$ by ${ }^{\hat{w}}: R_{\alpha} \mapsto R_{\hat{w}(\alpha)}$.

Definition 3.1. Let $\mathcal{I}$ be the ideal in $\mathcal{T}$ which is generated by all the elements of the form for $i \neq j \in I$, and $\hat{w} \in \widehat{W}$ :

$$
{ }^{\hat{w}}(\underbrace{R_{\alpha_{i}} \otimes R_{r_{i} \alpha_{j}} \otimes R_{r_{i} r_{j} \alpha_{i}} \otimes \cdots}_{m_{i j} \text { factors }})-{ }^{\hat{w}}(\underbrace{R_{\alpha_{j}} \otimes R_{r_{j} \alpha_{i}} \otimes R_{r_{j} r_{i} \alpha_{j}} \otimes \cdots}_{m_{i j} \text { factors }}) .
$$

The root algebra $\widetilde{\mathcal{R}}$ is $\mathcal{T} / \mathcal{I} .\left\{R_{\alpha} \mid \alpha \in \Delta^{r e}\right\}$ are called the $R$-matrices.

Because of the $\widehat{W}$-invariance of $\mathcal{I}$, the action of $\widehat{W}$ is induced on $\widetilde{\mathcal{R}}$. For simplicity, we write products in $\widetilde{\mathcal{R}}$ in the usual way for associative algebras.

Theorem 3.2. $\quad$ 1. There exists a unique set $\left\{R_{\hat{w}} \mid \hat{w} \in \widehat{W}\right\} \subset \widetilde{\mathcal{R}}$ satisfying the relations:

$$
R_{v w}=R_{v}{ }^{v} R_{w}, \quad R_{r_{i}}=R_{\alpha_{i}} \quad(i \in I), \quad R_{\omega}=1
$$

where $\omega \in \Omega, v, w \in \widehat{W}$ and $\ell(v w)=\ell(v)+\ell(w)$.

2. We have the $R$-matrix for $\hat{w} \in \widehat{W}$ and its arbitrary reduced decomposition $\hat{w}=w \omega=$ $r_{i_{1}} \ldots r_{i_{\ell}} \omega$ as

$$
R_{\hat{w}}=R_{\alpha^{1}} \ldots R_{\alpha^{\ell}}, \quad \alpha^{1}=\alpha_{i_{1}}, \quad \alpha^{2}=r_{i_{1}}\left(\alpha_{i_{2}}\right), \quad \ldots, \quad \alpha^{\ell}=w r_{i_{\ell}}\left(\alpha_{i_{\ell}}\right) \in \Delta_{\hat{w}} .
$$

Instead of the original root algebra, we use the following extension, where $\widetilde{\mathcal{R}}$ is combined with the translation group $T_{\widehat{M}}$ :

Definition 3.3. $\mathcal{R}:=\widetilde{\mathcal{R}} \rtimes T_{\widehat{M}}$ :

$$
\left(R t_{\lambda}\right)\left(R^{\prime} t_{\mu}\right)=R\left({ }^{t_{\lambda}} R^{\prime}\right) t_{\lambda+\mu}
$$

where $R, R^{\prime} \in \widetilde{\mathcal{R}}$ and $\lambda, \mu \in \widehat{M}$. 
We see that $\mathcal{R}$ is generated by $\left\{t_{\lambda_{i}}, R_{\alpha} \mid i \in \stackrel{\circ}{I}, \alpha \in \stackrel{\circ}{\Delta}\right\}$ if $A$ is not of type $A_{2 l}^{(2)}$ and $\left\{t_{\lambda_{i}}, R_{\alpha} \mid i \in \stackrel{\circ}{I}, \alpha \in \stackrel{\circ}{\Delta}, 2 \alpha-\delta \in \stackrel{\circ}{\Delta}\right\}$ if $A$ is of type $A_{2 l}^{(2)}$.

Theorem 3.4. The subalgebra $\mathcal{S} \subset \mathcal{R}$ generated by $\left\{Y^{\lambda}:=R_{t_{\lambda}} t_{\lambda} \mid \lambda \in \widehat{M}_{-}\right\}$forms a commutative algebra and is generated by $\left\{Y^{-\lambda_{i}} \mid i \in \stackrel{\circ}{I}\right\}$.

Proof. It is straightforward by the formulas (2.17) and Definition 3.3.

\section{Affine Root Systems of Rank 3}

There are six types of affine root systems of rank 3 . We denote $\alpha=\alpha_{1}$ and $\beta=\alpha_{2}$ where $\left|\alpha_{1}\right| \geq\left|\alpha_{2}\right|$, and $\lambda=\lambda_{1}$ and $\mu=\lambda_{2}$, respectively. We have the following systems:

$$
\begin{aligned}
& A_{2}^{(1)} \text {-type } \quad Y^{-\lambda}=R_{\alpha} R_{\alpha+\beta} t_{-\lambda}, \\
& Y^{-\mu}=R_{\beta} R_{\alpha+\beta} t_{-\mu}, \\
& C_{2}^{(1)} \text {-type } \quad Y^{-\lambda}=R_{\alpha} R_{\alpha+\beta} R_{\alpha+2 \beta} t_{-\lambda}, \\
& Y^{-\mu}=R_{\beta} R_{\alpha+2 \beta} R_{\alpha+\beta} R_{\alpha+2 \beta+\delta} t_{-\mu}, \\
& G_{2}^{(1)} \text {-type } \quad Y^{-\lambda}=R_{\alpha} R_{\alpha+\beta} R_{2 \alpha+3 \beta} R_{\alpha+2 \beta} R_{\alpha+3 \beta} R_{2 \alpha+3 \beta+\delta} t_{-\lambda} \text {, } \\
& Y^{-\mu}=R_{\beta} R_{\alpha+3 \beta} R_{\alpha+2 \beta} R_{2 \alpha+3 \beta} R_{\alpha+\beta} \\
& R_{\alpha+3 \beta+\delta} R_{2 \alpha+3 \beta+\delta} R_{\alpha+2 \beta+\delta} R_{\alpha+3 \beta+2 \delta} R_{2 \alpha+3 \beta+2 \delta} t_{-\mu}, \\
& A_{4}^{(2)} \text {-type } \quad Y^{-\lambda}=R_{\alpha} R_{\alpha+\beta} R_{\alpha+2 \beta} R_{\frac{1}{2} \alpha+\frac{1}{2} \delta} R_{\alpha+\beta+\delta} R_{\frac{1}{2} \alpha+\beta+\frac{1}{2} \delta} t_{-\lambda} \text {, } \\
& Y^{-\mu}=R_{\beta} R_{\alpha+2 \beta} R_{\alpha+\beta} R_{\frac{1}{2} \alpha+\beta+\frac{1}{2} \delta} t_{-\mu}, \\
& D_{3}^{(2)} \text {-type } \quad Y^{-\lambda}=R_{\alpha} R_{\alpha+\beta} R_{\alpha+2 \beta} R_{\alpha+\beta+\delta} t_{-\lambda} \text {, } \\
& Y^{-\mu}=R_{\beta} R_{\alpha+2 \beta} R_{\alpha+\beta} t_{-\mu},
\end{aligned}
$$




$$
\begin{aligned}
Y_{4}^{(3)} \text {-type }= & R_{\alpha} R_{\alpha+\beta} R_{2 \alpha+3 \beta} R_{\alpha+2 \beta} \\
& R_{\alpha+3 \beta} R_{\alpha+\beta+\delta} R_{\alpha+2 \beta+\delta} R_{2 \alpha+3 \beta+3 \delta} R_{\alpha+\beta+2 \delta} R_{\alpha+2 \beta+2 \delta} t_{-\lambda}, \\
Y^{-\mu}= & R_{\alpha} R_{\alpha+3 \beta} R_{\alpha+2 \beta} R_{2 \alpha+3 \beta} R_{\alpha+\beta} R_{\alpha+2 \beta+\delta} t_{-\mu} .
\end{aligned}
$$

\section{$5 \quad$ Elliptic $R$-matrices}

For $\alpha \in \Delta^{r e}$, let $\mu_{\alpha} \in \mathbb{C}$ be $\widehat{W}$-invariant constants: $\mu_{\hat{w}(\alpha)}=\mu_{\alpha}$ for $\hat{w} \in \widehat{W}$. Let $Y:=$ $\{h \in \mathfrak{h} \mid \operatorname{Re}\langle\delta, h\rangle>0\}$ and let $\mathcal{M}$ be the set of meromorphic functions on $Y$. We define an action of $w=\stackrel{\circ}{w} t_{\lambda} \in \widehat{W}$ on $\mathcal{M}$ as $(w f)(h):=f\left(t_{-\lambda}^{\iota} \stackrel{\circ}{w^{-1}}(h)\right)$.

Fix $\kappa \in \mathbb{C}$ and $\xi \in \stackrel{\circ}{\mathfrak{h}}^{*}$. We define $\hat{R}_{\alpha} \in \operatorname{End}_{\mathbb{C}} \mathcal{M}$ for $\alpha \in \Delta^{r e}$ by

$$
\hat{R}_{\alpha}:=H_{\alpha}\left(\mu_{\alpha}\right)-H_{\alpha}\left(\left\langle\xi, \alpha^{\vee}\right\rangle\right) r_{\alpha}
$$

with the following function (see the definitions in Appendix):

$$
H_{\alpha}(\nu):=\frac{\vartheta^{1}\left(-\gamma_{\alpha} \mu_{\alpha} \delta ; \gamma_{\alpha}\right)}{\vartheta^{1 \prime}\left(0 ; \gamma_{\alpha}\right)} \sigma_{\gamma_{\alpha} \nu}\left(\iota(\alpha) ; \gamma_{\alpha}\right)
$$

Theorem 5.1. The map $\pi: R_{\alpha} \mapsto \hat{R}_{\alpha}, t_{\lambda} \mapsto t_{\lambda}$ induces a homomorphism from $\mathcal{R}$ to End $\mathbb{C}_{\mathbb{C}} \mathcal{M}$. These R-matrices satisfy the unitarity

$$
\hat{R}_{\alpha} \hat{R}_{-\alpha}=\left(\frac{\vartheta^{1}\left(-\gamma_{\alpha} \mu_{\alpha} \delta ; \gamma_{\alpha}\right)}{\vartheta^{1 \prime}\left(0 ; \gamma_{\alpha}\right)}\right)^{2}\left(\wp^{0}\left(\gamma_{\alpha} \mu_{\alpha} \delta ; \gamma_{\alpha}\right)-\wp^{0}\left(\gamma_{\alpha}\left\langle\xi, \alpha^{\vee}\right\rangle \delta ; \gamma_{\alpha}\right)\right) I d_{\mathcal{M}}
$$

Besides the above representation, we have more general forms that depend on the relation among $Q, Q^{\vee}, M$. For $\alpha \in \Delta^{r e}$, let

$$
N_{\alpha}:=\left\{\begin{array}{l|l}
\phi_{\alpha}^{j}:=\left(m_{\alpha}, n_{\alpha}\right) \in \mathbb{R}_{>0}^{2} & \begin{array}{l}
\overline{\alpha^{\vee}} \in m_{\alpha} \stackrel{\circ}{Q}^{\vee}, \quad m_{\alpha}\left\langle\alpha, \stackrel{\circ}{Q^{\vee}}\right\rangle \subset \mathbb{Z}, \\
n_{\alpha} \gamma_{\alpha} \overline{\alpha^{\vee}} \in m_{\alpha} M, m_{\alpha}(M \mid \alpha) \subset n_{\alpha} \gamma_{\alpha} \mathbb{Z}
\end{array}
\end{array}\right\} .
$$

This condition is required when the root algebra acts on the vector space spanned by theta functions (Proposition [7.1) and is an elliptic analogue in the representation of the Hecke algebras [24].

We enumerate the set $N_{\alpha}$ as 


\begin{tabular}{|l||c|c|c|c|}
\hline & $\phi_{\alpha}^{1}$ & $\phi_{\alpha}^{2}$ & $\phi_{\alpha}^{3}$ & $\phi_{\alpha}^{4}$ \\
\hline \hline$A$ is of type $C_{l}^{(1)}$ and $\alpha$ is long & $(1,1)$ & $(1,2)$ & $(1 / 2,1)$ & $(1 / 2,1 / 2)$ \\
$A$ is of type $A_{2 l-1}^{(2)}$ and $\alpha$ is long & $(1,1)$ & & & $(1 / 2,1 / 2)$ \\
$A$ is of type $D_{l+1}^{(2)}$ and $\alpha$ is short & $(1,1)$ & $(1,2)$ & & \\
$A$ is of type $A_{2 l}^{(2)}$ and $\alpha$ is short & $(2,1)$ & $(2,2)$ & $(1,1)$ & $(1,1 / 2)$ \\
$A$ is of type $A_{2 l}^{(2)}$ and $\alpha$ is long & $(1,1 / 2)$ & $(1,1)$ & $(1 / 2,1 / 2)$ & $(1 / 2,1 / 4)$ \\
otherwise & $(1,1)$ & & & \\
\hline
\end{tabular}

Here we have numbered the elements of $N_{\alpha}$ for later convenience. Let $\zeta_{\alpha}^{j} \in \mathbb{C}$ for $1 \leq j \leq 4$ $\widehat{W}$-invariant constants. If $\phi_{\alpha}^{j} \notin N_{\alpha}$, set $\zeta_{\alpha}^{j}=0$. In place of (5.2), we define

$$
H_{\alpha}(\nu):=\sum_{\phi_{\alpha}^{j}=\left(m_{\alpha}, n_{\alpha}\right) \in N_{\alpha}} \zeta_{\alpha}^{j} \frac{\vartheta^{1}\left(-n_{\alpha} \gamma_{\alpha} \mu_{\alpha} \delta / m_{\alpha} ; n_{\alpha} \gamma_{\alpha}\right)}{\vartheta^{1 \prime}\left(0 ; n_{\alpha} \gamma_{\alpha}\right)} \sigma_{n_{\alpha} \gamma_{\alpha} \nu / m_{\alpha}}\left(\iota\left(m_{\alpha} \alpha\right) ; n_{\alpha} \gamma_{\alpha}\right) \text {. }
$$

Then we have a more general representation of $\mathcal{R}$ including Theorem 5.1.

Theorem 5.2. The map $\pi$ in Theorem 5.1 with (5.5) induces a homomorphism from $\mathcal{R}$ to $E n d_{\mathbb{C}} \mathcal{M}$. These R-matrices satisfy the unitarity

$$
\hat{R}_{\alpha} \hat{R}_{-\alpha}=u_{\alpha}(\delta) I d_{\mathcal{M}}
$$

where $u_{\alpha}(\delta)$ depends only on $\delta$ and vanishes if $\left\langle\xi, \alpha^{\vee}\right\rangle= \pm \mu_{\alpha}$.

More precisely, we have $u_{\alpha}(\delta)=\left(\left(p_{1} \cdot \zeta_{a}\right)^{2},\left(p_{2} \cdot \zeta_{a}\right)^{2},\left(p_{3} \cdot \zeta_{a}\right)^{2},\left(p_{4} \cdot \zeta_{a}\right)^{2}\right) \cdot S \cdot d_{\alpha}$,

$$
\begin{aligned}
& S=\frac{1}{4}\left(\begin{array}{cccc}
1 & 0 & 0 & 0 \\
-1 & 0 & 0 & 1 \\
-1 & 4 & 0 & 0 \\
1 & -4 & 4 & -1
\end{array}\right), \quad \zeta_{\alpha}=\left(\begin{array}{c}
\tilde{\zeta}_{\alpha}^{1} \\
\tilde{\zeta}_{\alpha}^{2} \\
\tilde{\zeta}_{\alpha}^{3} \\
\tilde{\zeta}_{\alpha}^{4}
\end{array}\right), \quad d_{\alpha}=\left(\begin{array}{c}
d_{\alpha}^{1} \\
d_{\alpha}^{2} \\
d_{\alpha}^{3} \\
d_{\alpha}^{4}
\end{array}\right), \\
& p_{1}=(2,1,1,2), \quad p_{2}=(0,0,1,2), \quad p_{3}=(0,1,1,0), \quad p_{4}=(0,0,1,0), \\
& \tilde{\zeta}_{\alpha}^{j}=\zeta_{\alpha}^{j} \frac{\vartheta^{1}\left(-n_{\alpha} \gamma_{\alpha} \mu_{\alpha} \delta / m_{\alpha} ; n_{\alpha} \gamma_{\alpha}\right)}{\vartheta^{1 \prime}\left(0 ; n_{\alpha} \gamma_{\alpha}\right)}, \\
& d_{\alpha}^{j}=\wp^{0}\left(n_{\alpha} \gamma_{\alpha} \mu_{\alpha} \delta / m_{\alpha} ; n_{\alpha} \gamma_{\alpha}\right)-\wp^{0}\left(n_{\alpha} \gamma_{\alpha}\left\langle\xi, \alpha^{\vee}\right\rangle \delta / m_{\alpha} ; n_{\alpha} \gamma_{\alpha}\right)
\end{aligned}
$$

Proof. We can verify the relations (3.1) case-by-case, by a direct substitution of (5.5); for details, see [18, 19, 30].

We employ these operators even for the affine root systems of rank 2, though they do not have any Coxeter relations. 
We shall clarify some properties of the operators $\hat{Y}^{\lambda}=\pi\left(Y^{\lambda}\right)$.

Lemma 5.3. The $R$-matrices $\hat{R}$ satisfy the following relations:

$$
\begin{aligned}
& \hat{R}_{-\alpha_{j}} \hat{R}_{t_{-\lambda_{i}}}={ }^{r_{j}} \hat{R}_{t_{-\lambda_{i}}} \hat{R}_{-\alpha_{j}}, \quad \text { for } j \neq i ; \\
& \hat{R}_{t_{-\lambda_{i}}}=\hat{R}_{\alpha_{i}} \mathscr{R},
\end{aligned}
$$

where $\mathscr{R}$ is a product of some $R$-matrices.

Proof. Combining (2.17), the unitarity (5.6), and an equality $r_{j} t_{-\lambda_{i}}=t_{-\lambda_{i}} r_{j}$, we obtain (5.7) for generic $\xi$ and thus for all $\xi \in \stackrel{\circ}{\mathfrak{h}}^{*}$. The form (5.8) is due to the fact that $\ell\left(r_{i} t_{-\lambda_{i}}\right)=$ $\ell\left(t_{-\lambda_{i}}\right)-1$ implies the exchange condition [2], $t_{-\lambda_{i}}=r_{i_{1}} \ldots r_{i_{\ell}} \omega=r_{i} r_{i_{1}} \ldots r_{i_{m-1}} r_{i_{m+1}} \ldots r_{i_{\ell}} \omega$ for some $m$.

If the parameter $\xi$ satisfies $\left\langle\xi, \alpha_{i}^{\vee}\right\rangle=-\mu_{-\alpha_{i}}$, then the $R$-matrix $\hat{R}_{-\alpha_{i}}$ reduces to the form, $\hat{R}_{-\alpha_{i}}=2 H_{-\alpha}\left(\mu_{-\alpha}\right) P_{i}^{(-)}$where $P_{i}^{(-)}$is the antisymmetric projection $\frac{1}{2}\left(1-r_{i}\right)$. Let

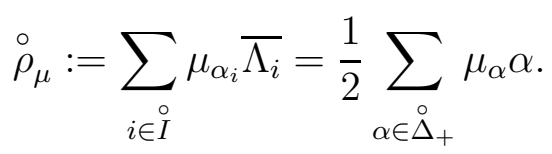

From these properties, we have the following theorem:

Theorem 5.4. Let $\mathcal{V}:=\stackrel{\circ}{W}^{W}$, the $\stackrel{\circ}{W}$-invariant subspace of $\mathcal{M}$ and let $\xi=-\stackrel{\circ}{\rho}_{\mu}$. Then $\hat{Y}^{\lambda} \in \operatorname{End}_{\mathbb{C}} \mathcal{V}$.

Proof. It is sufficient to check it for the generators $\hat{Y}^{-\lambda_{i}}$. By Lemma 5.3, we see that $\left.\hat{R}_{-\alpha_{j}} \hat{Y}^{-\lambda_{i}}\right|_{\mathcal{V}}=0$, for $j \neq i$ by (5.7), and for $j=i$ by (5.8) noting that the unitarity (5.6) vanishes. Hence $\left.\hat{Y}^{-\lambda_{i}}\right|_{\mathcal{V}}=\left.r_{j} \hat{Y}^{-\lambda_{i}}\right|_{\mathcal{V}}$ for all $j \in \stackrel{\circ}{I}$.

The symbol $Y^{\lambda}$ is adopted since in a certain limit, it reduces to the same one up to a constant factor as in the affine Hecke algebras, where $Y^{\lambda}$ is defined for all $\lambda \in \widehat{M}$. We remark that $\hat{Y}^{\lambda}$ has the inverse in End $\mathbb{C} \mathcal{M}$ for generic $\xi \in \stackrel{\circ}{\mathfrak{h}}^{*}$, but loses its inverse when $\xi=-\stackrel{\circ}{\rho}_{\mu}$. 


\section{$6 \quad$ Elliptic Difference Operators}

In this section, we calculate the explicit forms of the operators $\hat{Y}^{\lambda}$ for some $\lambda$ on the space $\mathcal{V}$. Throughout this section, we fix $\xi=-\stackrel{\circ}{\rho}_{\mu}$.

Theorem 6.1. Let $(-\lambda)$ be minuscule. Then we have

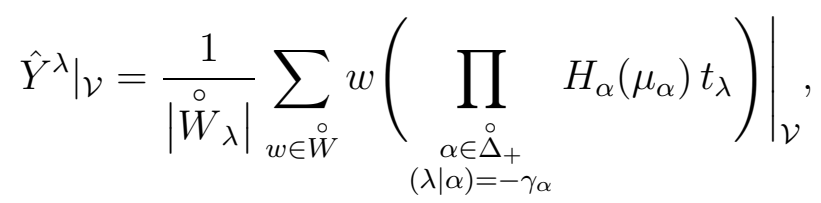

where $\stackrel{\circ}{W}_{\lambda}$ is the stabilizer of $\lambda$ in $\stackrel{\circ}{W}$.

Proof. First notice that $R_{t_{\lambda}}$ consists of nonaffine $R$-matrices, $R_{\alpha}$ for $\alpha \in \stackrel{\circ}{\Delta}_{+}$, because $(-\lambda)$ is minuscule. Substituting the $R$-matrices (5.1) into $\hat{Y}^{\lambda}$ and expanding them, we see that every term includes a translation operator of the form $t_{w(\lambda)} w$, where

$$
\begin{aligned}
& w=r_{\alpha\{p\}} \ldots r_{\alpha\{1\}} \in \stackrel{\circ}{W}, \\
& \alpha\{q\}:=\alpha^{m_{q}}, \quad 1 \leq m_{p}<m_{p-1}<\ldots<m_{2}<m_{1} \leq \ell\left(t_{\lambda}\right) .
\end{aligned}
$$

Let us show that $w(\lambda)=\lambda$ implies $w=i d$. Suppose $w(\lambda)=\lambda$ and $w \neq i d$, then we have $\ell\left(w t_{\lambda}\right)=\ell\left(t_{\lambda} w\right)$. From (2.17), $\ell\left(t_{\lambda} w\right)=\ell\left(t_{\lambda}\right)+\ell(w)>\ell\left(t_{\lambda}\right)$ while $\ell\left(w t_{\lambda}\right)<\ell\left(t_{\lambda}\right)$ by a direct calculation, which leads to a contradiction. This implies that the term including $t_{\lambda} w, w \in \stackrel{\circ}{W}$ appears if and only if $w=i d$. The coefficient of this term can be easily calculated,

$$
\prod_{\substack{\alpha \in \dot{\Delta}_{+} \\(\lambda \mid \alpha)=-\gamma_{\alpha}}} H_{\alpha}\left(\mu_{\alpha}\right) .
$$

The $\stackrel{\circ}{W}$-invariance of the operator $\hat{Y}^{\lambda}$ yields the form (6.1).

It is worth noting that as in the trigonometric case [26], we can rewrite $\hat{Y}^{\lambda}$ in a simply laced root system as follows:

$$
\left.\hat{Y}^{\lambda}\right|_{\mathcal{V}}=\left.\frac{1}{\left|\stackrel{\circ}{W}_{\lambda}\right|} \sum_{w \in \stackrel{\circ}{W}} \frac{\left(t_{-\mu w \lambda / \kappa} A_{\rho}\right)}{A_{\rho}} t_{w \lambda}\right|_{\mathcal{V}},
$$


where we have set $\mu=\mu_{\alpha}$ and $\zeta_{\alpha}^{1}=1$.

In general, it is complicated and difficult to compute the explicit forms of the operators when $\lambda$ is not minuscule. It is the case even in the framework of the affine Hecke algebras. There is no minuscule weight available in the root systems of type $E_{8}^{(1)}, F_{4}^{(1)}, G_{2}^{(1)}, A_{2 l}^{(2)}, E_{6}^{(2)}$ and $D_{4}^{(3)}$. However, every root system possesses a "quasi-minuscule" weight $\nu\left(\theta^{\vee}\right)$ in the sense of the following properties:

Lemma 6.2. 1. $\Delta_{t_{-\nu(\theta} \vee}=\Delta_{r_{\theta}} \cup\left\{a_{0}^{-1}(\delta+\theta)\right\}$.

2. $\left(\nu\left(\theta^{\vee}\right) \mid \alpha\right)=0$ or $\gamma_{\alpha}$ for $\alpha \in \stackrel{\circ}{\Delta}_{+}, \alpha \neq \theta$, and $\left(\nu\left(\theta^{\vee}\right) \mid \theta\right)=2$.

3. $\nu\left(\theta^{\vee}\right)=\lambda_{i}$ where $\alpha_{i}$ is the unique vertex connected to $\alpha_{0}$ if $A$ is not of type $A_{l}^{(1)}$.

Proof. We see that $r_{\theta} \alpha_{0}=r_{\theta}\left(a_{0}^{-1}(\delta-\theta)\right)=a_{0}^{-1}(\delta+\theta) \in \Delta_{+}^{r e}$, which implies the first statement due to the expression $r_{\theta} r_{0}=t_{-\nu\left(\theta^{\vee}\right)}$. The second statement is immediate from the first and (2.17). Since $\left\langle\nu\left(\theta^{\vee}\right), \alpha_{i}^{\vee}\right\rangle=\left\langle a_{0}^{-1} \theta, \alpha_{i}^{\vee}\right\rangle=\left\langle a_{0}^{-1} \delta-\alpha_{0}, \alpha_{i}^{\vee}\right\rangle=-\left\langle\alpha_{0}, \alpha_{i}^{\vee}\right\rangle$, we have $\nu\left(\theta^{\vee}\right)=-\sum_{i \in I}\left\langle\alpha_{0}, \alpha_{i}^{\vee}\right\rangle \overline{\Lambda_{i}}$. Then the last statement follows from the tables in [2, 16].

Since in the root system of type $A_{l}^{(1)}$, every $\lambda_{i}$ is minuscule, we have the explicit form of $\hat{Y}^{-\nu\left(\theta^{\vee}\right)}$ by Theorem 6.1. So we concentrate on the other root systems. Fix $i$ as in Lemma 6.2 .

By the expression $t_{-\nu\left(\theta^{\vee}\right)}=r_{\theta} r_{0}$, we have $Y^{-\nu\left(\theta^{\vee}\right)}=R_{r_{\theta}} R_{a_{0}^{-1}(\theta+\delta)} t_{-\nu\left(\theta^{\vee}\right)}=R_{r_{\theta}} t_{-\nu\left(\theta^{\vee}\right)} R_{-\alpha_{0}}$. For the operator $\hat{Y}^{-\nu\left(\theta^{\vee}\right)}$, an analogous statement to Lemma 5.3 holds.

Lemma 6.3. The $R$-matrices $\hat{R}$ satisfy the following relations:

$$
\begin{aligned}
& \hat{R}_{-\alpha_{j}} \hat{R}_{r_{\theta}}={ }^{r_{j}} \hat{R}_{r_{\theta}} \hat{R}_{-\alpha_{j}}, \quad \text { for } j \neq i ; \\
& \hat{R}_{r_{\theta}}=\hat{R}_{\alpha_{i}} \mathscr{R},
\end{aligned}
$$

where $\mathscr{R}$ is a product of some $R$-matrices.

Proof. We have $r_{j} t_{-\nu\left(\theta^{\vee}\right)}=t_{-\nu\left(\theta^{\vee}\right)} r_{j}$ and $r_{j} r_{0}=r_{0} r_{j}$ for $j \neq i$, since $\alpha_{i}$ is the unique vertex connected to $\alpha_{0}$. Then $r_{j}$ and $r_{\theta}=t_{-\nu\left(\theta^{\vee}\right)} r_{0}$ commute, which implies $\ell\left(r_{j} r_{\theta}\right)=\ell\left(r_{\theta}\right)+1$ 
and thus (6.6). The form (6.7) follows from the fact that $\ell\left(r_{i} t_{-\nu\left(\theta^{\vee}\right)}\right)=\ell\left(t_{-\nu\left(\theta^{\vee}\right)}\right)-1$ implies $\ell\left(r_{i} r_{\theta}\right)=\ell\left(r_{\theta}\right)-1$ and the exchange condition.

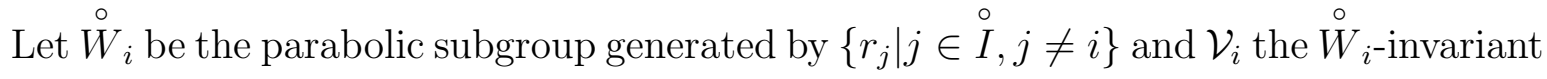
subspace of $\mathcal{M}$.

Lemma 6.4. The operator $\hat{R}_{r_{\theta}} t_{-\nu\left(\theta^{\vee}\right)}$ maps $\mathcal{V}_{i}$ to $\mathcal{V}$ and the operator $\hat{R}_{-\alpha_{0}}, \mathcal{V}$ to $\mathcal{V}_{i}$.

Proof. The former statement can be shown in the same way as Theorem 5.4, and the latter, directly.

\section{Theorem 6.5.}

$$
\begin{aligned}
& \left.\hat{Y}^{-\nu\left(\theta^{\vee}\right)}\right|_{\mathcal{V}}=
\end{aligned}
$$

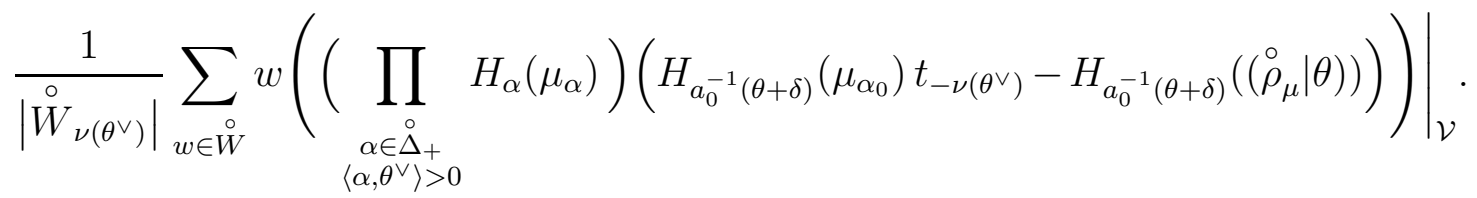

Proof. The explicit form of $\hat{R}_{r_{\theta}} t_{-\nu\left(\theta^{\vee}\right)}$ on $\mathcal{V}_{i}$ can be computed in a similar way to Theorem 6.1. Since $Y^{-\nu\left(\theta^{\vee}\right)}=R_{r_{\theta}} t_{-\nu\left(\theta^{\vee}\right)} R_{-\alpha_{0}}$, we obtain the form (6.8).

The operator (1.2) is actually (6.8) of type $A_{2 l}^{(2)}$, where the terms without translations are gathered by use of identities of the theta functions. In [20, 22], we calculated the explicit forms of $\left.\hat{Y}^{-\lambda_{j}}\right|_{\mathcal{V}}$ for all $j \in \stackrel{\circ}{I}$ in this root system. The operator (6.8) in the affine root systems of type $E_{8}^{(1)}, F_{4}^{(1)}, G_{2}^{(1)}$ and $A_{2 l}^{(2)}$ should be compared to the Macdonald(Koornwinder) operator $D_{\theta} \vee$ of type $E_{8}, F_{4}, G_{2}$ and $B C_{l}$ respectively, while the operator (6.1) in the rest root systems of type $X_{l}^{(1)}$ to $E_{\nu^{-1}\left(\lambda_{i}\right)}$ of type $X_{l}$ [23,25].

In order to investigate a general $\hat{Y}^{\lambda}$, let us define a partial order in $\widehat{M}_{-}$. We remark that this partial order is different from that in the affine Hecke algebras.

Definition 6.6. Let $\lambda, \lambda^{\prime} \in \widehat{M}_{-}$. We write $\lambda \succeq \lambda^{\prime}$ if $\ell\left(t_{\lambda}\right)>\ell\left(t_{\lambda^{\prime}}\right)$ or $\lambda=\lambda^{\prime}$. 
For an arbitrary weight $\lambda \in \widehat{M}_{-}$, we have the "leading term" of $\hat{Y}^{\lambda}$ with respective to the order $\succ$.

Theorem 6.7. Let $\lambda \in \widehat{M}_{-}$. Then we have

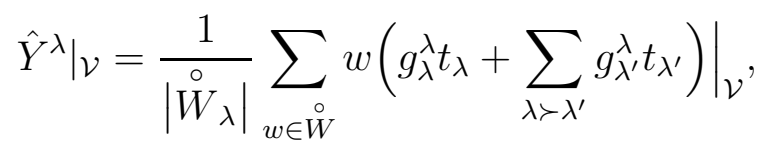

where $g_{\lambda^{\prime}}^{\lambda} \in \mathcal{M}$. Especially we have $g_{\lambda}^{\lambda}=\prod_{\alpha \in \Delta_{t_{\lambda}}} H_{\alpha}\left(\mu_{\alpha}\right)$.

Proof. Because $\hat{Y}^{\lambda}$ is $\stackrel{\circ}{W}$-invariant, it is sufficient to calculate the coefficients of the translations of antidominant weights. A translation $t_{\lambda^{\prime}}, \lambda^{\prime} \in \widehat{M}_{-}$in the expansion of $\hat{Y}^{\lambda}$ appears as $w t_{\lambda}=t_{\lambda^{\prime}} \stackrel{\circ}{w}$ where $\stackrel{\circ}{w} \in \stackrel{\circ}{W}$ and

$$
\begin{aligned}
& w=r_{\alpha\{p\}} \ldots r_{\alpha\{1\}} \in W \\
& \alpha\{q\}=\alpha^{m_{q}}, \quad 1 \leq m_{p}<m_{p-1}<\ldots<m_{2}<m_{1} \leq \ell\left(t_{\lambda}\right) .
\end{aligned}
$$

Then $\ell\left(t_{\lambda}\right) \geq \ell\left(w t_{\lambda}\right)=\ell\left(t_{\lambda^{\prime}} \stackrel{\circ}{w}\right)=\ell\left(t_{\lambda^{\prime}}\right)+\ell(\stackrel{\circ}{w})$, which implies $\ell\left(t_{\lambda}\right)>\ell\left(t_{\lambda^{\prime}}\right)$ if $w \neq i d$. Hence the expression (6.9).

Theorem 6.8 (cf. $[4)$. $\left\{\hat{Y}^{-\lambda_{i}} \mid i \in \stackrel{\circ}{I}\right\}$ are algebraically independent on $\mathcal{V}$.

Proof. Consider $Y=\sum_{\lambda} a_{\lambda} Y^{\lambda} \in \mathcal{S}$ with $a_{\lambda} \in \mathbb{C}$. Let $M_{Y}$ be the set of all the maximal antidominant weights in the expansion of $\hat{Y}$ on $\mathcal{V}$. Let $M_{Y}^{\prime}:=\cup_{\lambda \in M_{Y}}\left\{\lambda^{\prime} \in \widehat{M}_{-} \mid \lambda^{\prime} \preceq \lambda\right\}$. Then we have

$$
\left.\hat{Y}\right|_{\mathcal{V}}=\left.\sum_{\lambda \in M_{Y}} \sum_{\substack{\infty \\ w \in W}} w\left(a_{\lambda} g_{\lambda}^{\lambda} t_{\lambda}+\text { lower terms }\left(\lambda^{\prime} \prec \lambda\right)\right)\right|_{\mathcal{V}}
$$

Fix $\lambda \in M_{Y}$. There exists $h_{0} \in \mathfrak{h}$ such that

$$
\left\{w h_{0}-h_{0} \mid w \in \stackrel{\circ}{W}\right\} \cap\left\{\kappa^{-1}\left\langle\delta, h_{0}\right\rangle\left(\nu^{-1}\left(w \lambda^{\prime}\right)-\nu^{-1}\left(w^{\prime} \lambda^{\prime}\right)\right) \mid w, w^{\prime} \in \stackrel{\circ}{W}, \lambda^{\prime} \in M_{Y}^{\prime}\right\}=\emptyset
$$

and $g_{\lambda}^{\lambda}\left(h_{0}\right) \neq 0$. Suppose $\hat{Y} f=0$ for all $f \in \mathcal{V}$. Then $(\hat{Y} f)\left(h_{0}\right)=0$. Since $\left\{\left(t_{w \lambda^{\prime}} f\right)\left(h_{0}\right) \mid \lambda^{\prime} \in\right.$ $\left.M_{Y}^{\prime}, w \in \stackrel{\circ}{W}\right\}$ can be made arbitrary for suitable $f \in \mathcal{V}$, it follows that $a_{\lambda}=0$ and hence the result.

Corollary 6.9. $\mathcal{S} \simeq \mathbb{C}\left[T_{\widehat{M}_{-}}\right]$. 


\section{Action on Theta Functions of Level $k$}

The aim of this section is to show that the operators $\hat{Y}^{\lambda}$ in the previous sections act on $\left(\widetilde{T h^{k}}\right)^{\stackrel{\circ}{W}}$, the $\stackrel{\circ}{W}$-invariant space of the theta functions of level $k$ or the space of the characters. To be more precise, we identify $\hat{Y}^{\lambda}$ with an operator on $\left(\widetilde{T h}^{k}\right)^{W}$ by restricting the domain. We regard this space both as a $\mathbb{C}$-vector space and as an $\mathscr{O}$-module. The basic idea is from [9, 19], where the matrix elements of Belavin's $\mathbb{Z}_{k}$-symmetric elliptic $R$-matrix and associated $K$-matrices are calculated. Now it is turned out that they treat the elliptic difference operators of type $A_{1}^{(1)}$ or $A_{2}^{(2)}$.

First let us outline our strategy. Since the representation $\pi$ in Theorem 5.2 does not preserve $\widetilde{T h}^{k}$ for general $\xi \in \mathfrak{h}^{*}$, we introduce another representation $\bar{\pi}$ which always preserve this space. The images of $\mathcal{S}$ by $\pi$ and $\bar{\pi}$ coincide when we set $\xi=-\stackrel{\circ}{\rho}_{\mu}$. As was shown, $\pi(\mathcal{S})$ at this value preserves $\stackrel{\circ}{W}$-invariant subspace, so does $\bar{\pi}(\mathcal{S})$. On the other hand, $\bar{\pi}(\mathcal{S})$ preserves $\widetilde{T h}{ }^{k}$ by construction, so does $\pi(\mathcal{S})$. Therefore we can deduce that $\pi(\mathcal{S})=\bar{\pi}(\mathcal{S})$ acts on $\left(\widetilde{T h^{k}}\right)^{\stackrel{\circ}{W}}$.

Let $h_{\mu}^{\vee}:=\left(\stackrel{\circ}{\rho}_{\mu} \mid \theta\right)+\mu_{\alpha_{0}}=\sum_{i \in I} \mu_{\alpha_{i}} a_{i}^{\vee}$ and $\Xi:=\frac{\xi+\stackrel{\circ}{\rho}_{\mu}}{h_{\mu}^{\vee}}$. Throughout this section, we fix $\kappa=\frac{h_{\mu}^{\vee}}{k}$ though some of the following statements do not require this condition.

We extend the action of $t_{\lambda}$ on $\mathcal{M}$ for arbitrary $\lambda \in \stackrel{\circ}{h}^{*}$ by $\left(t_{\lambda} f\right)(h):=f\left(t_{-\lambda}^{\iota} h\right)$. Let $\bar{R}_{\alpha} \in$ End $_{\mathbb{C}} \mathcal{M}$ be defined by

$$
\bar{R}_{\alpha}:=t_{\epsilon_{\alpha}^{1}} \hat{R}_{\bar{\alpha}} t_{\epsilon_{\alpha}^{2}}
$$

where $\epsilon_{\alpha}^{1}:=\frac{1}{h_{\mu}^{(}}\left(-\frac{1}{2} \mu_{\alpha} \bar{\alpha}-\xi+\eta_{\alpha}\right), \epsilon_{\alpha}^{2}:=\frac{1}{h_{\mu}^{(}}\left(-\frac{1}{2} \mu_{\alpha} \bar{\alpha}+\xi-\eta_{\alpha}\right)$, and $\eta_{\alpha} \in \stackrel{\circ}{\mathfrak{h}^{*}}$ is taken arbitrary such that $\left\langle\eta_{\alpha}, \overline{\alpha^{v}}\right\rangle=0$. Then $\bar{R}_{\alpha}$ does not depend on the choice of $\eta_{\alpha}$ and thus is well-defined. According to our plan, we show that this operator acts on $\widetilde{T h^{k}}$.

Proposition 7.1. For arbitrary $\xi \in \stackrel{\circ}{\mathfrak{h}}^{*}, \bar{R}_{\alpha} \in \operatorname{End}_{\mathscr{O}}\left(\widetilde{T h}^{k}\right)$.

Proof. We note that

$$
t_{\alpha}^{\iota} t_{\beta}=t_{\beta} t_{\alpha}^{\iota} f^{(\alpha \mid \beta)}
$$


where $\alpha \in \widehat{M}, \beta \in M$ and $f(\lambda):=\lambda-\kappa\langle\lambda, K\rangle \delta$ [5]. By using this relation and the condition (5.4), we can check the behavior under the action of the Heisenberg group (see the Appendix) and the holomorphy on the domain $Y$. Then we see $\bar{R}_{\alpha} \in \operatorname{End}_{\mathbb{C}}\left(\widetilde{T h^{k}}\right)$. Since $\widehat{W}$ fixes $\delta$, we have the proof.

Here we shall make crucial steps to the main statement.

Lemma 7.2. Let $\hat{w}=r_{i_{1}} \ldots r_{i_{\ell}} \omega \in \widehat{W}$ be a reduced expression. Let

$$
\eta_{n}:=-\stackrel{\circ}{\rho}_{\mu}+\sum_{m=1}^{n-1} \nu_{m} \overline{\alpha^{m}}+\frac{1}{2} \nu_{n} \overline{\alpha^{n}},
$$

where $\Delta_{\hat{w}}=\left\{\alpha^{1}=\alpha_{i_{1}}, \alpha^{2}=r_{i_{1}}\left(\alpha_{i_{2}}\right), \ldots, \alpha^{\ell}=w r_{i_{\ell}}\left(\alpha_{i_{\ell}}\right)\right\}$,

$$
\nu_{n}:= \begin{cases}\mu_{n}, & \text { if } \alpha_{i_{n}} \neq \alpha_{0} ; \\ -\left(\stackrel{\circ}{\rho}_{\mu} \mid \theta\right), & \text { if } \alpha_{i_{n}}=\alpha_{0},\end{cases}
$$

and $\mu_{n}:=\mu_{\alpha^{n}}$. Then $\left\langle\eta_{n}, \overline{\left(\alpha^{n}\right)^{\vee}}\right\rangle=0$.

Proof. First observe that if $\alpha^{n}=r_{i_{1}} \cdots r_{i_{n-1}} \alpha_{i_{n}}$, then $\overline{\alpha^{n}}=\bar{r}_{i_{1}} \cdots \bar{r}_{i_{n-1}} \overline{\alpha_{i_{n}}}$ and $\overline{\left(\alpha^{n}\right)^{\vee}}=$ $\bar{r}_{i_{1}} \cdots \bar{r}_{i_{n-1}} \overline{\alpha_{i_{n}}^{\vee}}$, where $\bar{r}_{i}:=r_{i}$ for $i \neq 0$ and $\bar{r}_{0}:=r_{\theta}$.

$$
\begin{aligned}
\left\langle-\stackrel{\circ}{\rho}_{\mu}, \overline{\left(\alpha^{n}\right)^{\vee}}\right\rangle & =\left\langle-\stackrel{\circ}{\rho}_{\mu}, \bar{r}_{i_{1}} \cdots \bar{r}_{i_{n-1}} \overline{\alpha_{i_{n}}^{\vee}}\right\rangle \\
& =\left\langle-\stackrel{\circ}{\rho}_{\mu}+\nu_{1} \overline{\alpha^{1}}, \bar{r}_{i_{2}} \cdots \bar{r}_{i_{n-1}} \overline{\alpha_{i_{n}}^{\vee}}\right\rangle \\
& =\left\langle-\stackrel{\circ}{\rho}_{\mu}, \bar{r}_{i_{2}} \cdots \bar{r}_{i_{n-1}} \overline{\alpha_{i_{n}}^{\vee}}\right\rangle-\nu_{1}\left\langle\overline{\alpha^{1}}, \overline{\left(\alpha^{n}\right)^{\vee}}\right\rangle \\
& \vdots \\
& =-\sum_{m=1}^{n-1} \nu_{m}\left\langle\overline{\alpha^{m}}, \overline{\left(\alpha^{n}\right)^{\vee}}\right\rangle-\nu_{n} .
\end{aligned}
$$

Then we have

$$
\left\langle-\stackrel{\circ}{\rho}_{\mu}+\sum_{m=1}^{n-1} \nu_{m} \overline{\alpha^{m}}+\frac{1}{2} \nu_{n} \overline{\alpha^{n}}, \overline{\left(\alpha^{n}\right)^{\vee}}\right\rangle=0 .
$$


Proposition 7.3. Let $\hat{w}=r_{i_{1}} \cdots r_{i_{\ell}} \omega \in \widehat{W}$ be a reduced expression. Then

$$
\bar{R}_{\alpha^{1}} \bar{R}_{\alpha^{2}} \cdots \bar{R}_{\alpha^{\ell}}=t_{-\Xi} \hat{R}_{\alpha^{1}} \hat{R}_{\alpha^{2}} \cdots \hat{R}_{\alpha^{\ell}} t_{\lambda} t_{\Xi}
$$

where $\lambda=-\frac{1}{h_{\mu}^{\vee}} \sum_{n=1}^{\ell} \mu_{n} \overline{\alpha^{n}}=-\frac{1}{h_{\mu}^{\vee}} \sum_{\alpha \in \Delta_{\hat{w}}} \mu_{\alpha} \bar{\alpha}$.

Proof. We set $\eta_{\alpha^{n}}=\eta_{n}$ obtained in Lemma 7.2 and set

$$
\begin{aligned}
& \epsilon_{0}:=\epsilon_{\alpha^{1}}^{1}=-\Xi+\frac{1}{2 h_{\mu}^{\vee}}\left(\nu_{1}-\mu_{1}\right) \overline{\alpha^{1}}, \\
& \epsilon_{n}:=\epsilon_{\alpha^{n}}^{2}+\epsilon_{\alpha^{n+1}}^{1}=\frac{1}{h_{\mu}^{\vee}}\left(\frac{1}{2}\left(\nu_{n}-\mu_{n}\right) \overline{\alpha^{n}}+\frac{1}{2}\left(\nu_{n+1}-\mu_{n+1}\right) \overline{\alpha^{n+1}}\right), \quad 1 \leq n \leq \ell-1, \\
& \epsilon_{\ell}:=\epsilon_{\alpha^{\ell}}^{2}=\Xi+\frac{1}{h_{\mu}^{\vee}}\left(-\sum_{m=1}^{\ell-1} \nu_{m} \overline{\alpha^{m}}-\frac{1}{2}\left(\nu_{\ell}+\mu_{\ell}\right) \overline{\alpha^{\ell}}\right) .
\end{aligned}
$$

Because $r_{i_{1}} \cdots r_{i_{\ell}} \omega$ is a reduced expression, we have for $1 \leq n \leq \ell-1$

$$
\epsilon_{n}= \begin{cases}-\frac{1}{2} \overline{\alpha^{n}}, & \text { if } \alpha_{i_{n}}=\alpha_{0} \\ -\frac{1}{2} \overline{\alpha^{n+1}}, & \text { if } \alpha_{i_{n+1}}=\alpha_{0} \\ 0, & \text { otherwise }\end{cases}
$$

Let $\bar{w}_{n}=\bar{r}_{i_{1}} \cdots \bar{r}_{i_{n}}$. If $\alpha_{i_{n}}=\alpha_{0}$, then $t_{\left(-\overline{\alpha^{n}} / 2\right)} \hat{R}_{\left(\bar{\alpha}^{n}\right)} t_{\left(-\overline{\alpha^{n}} / 2\right)}=\hat{R}_{\left(\bar{w}_{n-1} \alpha_{0}\right)} t_{\left(\bar{w}_{n-1} \nu\left(\theta^{\vee}\right)\right)}$ and if $\alpha_{i_{n}} \neq \alpha_{0}$, then $\hat{R}_{\left(\bar{\alpha}^{n}\right)}=\hat{R}_{\left(\bar{w}_{n-1} \alpha_{i_{n}}\right)}$. By using the identity

$$
\alpha^{n}=r_{i_{1}} \cdots r_{i_{n-1}} \alpha_{i_{n}}=\left(\prod_{\substack{m<n \\ \alpha_{i_{m}}=\alpha_{0}}} t_{\left(\bar{w}_{m-1} \nu\left(\theta^{\vee}\right)\right)}\right) \bar{w}_{n-1} \alpha_{i_{n}},
$$

we arrive at (7.7).

Apply this proposition to an element that has two reduced expressions of the form

$$
\hat{w}=\underbrace{r_{i} r_{j} r_{i} \ldots}_{m_{i j} \text { factors }}=\underbrace{r_{j} r_{i} r_{j} \ldots}_{m_{i j} \text { factors }}
$$

for $i \neq j \in I$. Then the relation

$$
\bar{R}_{\alpha_{i}} \bar{R}_{r_{i} \alpha_{j}} \bar{R}_{r_{i} r_{j} \alpha_{i}} \cdots=\bar{R}_{\alpha_{j}} \bar{R}_{r_{j} \alpha_{i}} \bar{R}_{r_{j} r_{i} \alpha_{j}} \cdots
$$

immediately follows. Regarding $w \Pi$ for $w \in \stackrel{\circ}{W}$, as a set of fundamental roots in Lemma 7.2 and Proposition 7.3, we have proved the following theorem: 
Theorem 7.4. The map $\bar{\pi}: R_{\alpha} \mapsto \bar{R}_{\alpha}, t_{\lambda} \mapsto I d_{\mathcal{M}}$ induces a homomorphism from $\mathcal{R}$ to $\operatorname{End}_{\mathbb{C}} \mathcal{M}$ and $\operatorname{End}_{\mathscr{O}}\left(\widetilde{T h^{k}}\right)$.

For $\lambda \in \widehat{M}_{-}$, we set $\bar{Y}^{\lambda}:=\bar{\pi}\left(Y^{\lambda}\right)=\bar{R}_{\alpha^{1}} \bar{R}_{\alpha^{2}} \cdots \bar{R}_{\alpha^{\ell}} \in \operatorname{End}_{\mathscr{O}}\left(\widetilde{T h^{k}}\right)$. Now we are in position to prove the main theorem fully stated as follows:

Theorem 7.5. Let $\kappa=\frac{h_{\mu}^{\vee}}{k}$ and $\xi=-\stackrel{\circ}{\rho}_{\mu}$. Then $\hat{Y}^{\lambda}=\bar{Y}^{\lambda} \in \operatorname{End}_{\mathscr{O}}\left(\left(\widetilde{T h^{k}}\right)^{W}\right)$.

By Proposition 7.3, we have already shown

$$
\bar{Y}^{\lambda}=t_{-\Xi} \hat{R}_{\alpha^{1}} \cdots \hat{R}_{\alpha^{\ell}} t_{\lambda^{\prime}} t_{\Xi}=t_{-\Xi} \hat{R}_{t_{\lambda}} t_{\lambda^{\prime}} t_{\Xi}
$$

where $\lambda^{\prime}=-\frac{1}{h_{\mu}^{\vee}} \sum_{n=1}^{\ell} \mu_{n} \overline{\alpha^{n}}=-\frac{1}{h_{\mu}^{\vee}} \sum_{\alpha \in \Delta_{t-\lambda}} \mu_{\alpha} \bar{\alpha}$. Since $\Xi=0$ if we set $\xi=-\stackrel{\circ}{\rho}_{\mu}$, we have only to show that $\lambda^{\prime}=\lambda$.

Due to the formulas (2.17), we have another description of $\lambda^{\prime}$ which can be regarded as an image of $\lambda$ by some linear map:

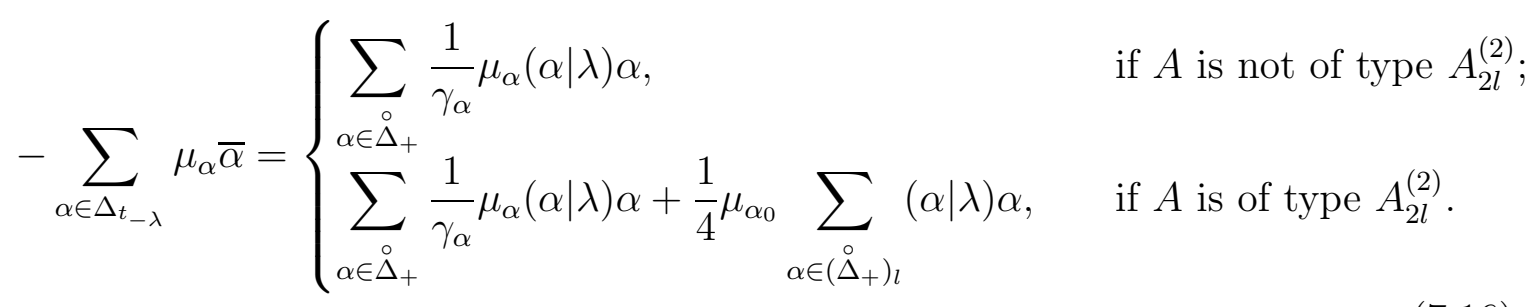

Lemma 7.6. Let $L: \stackrel{\circ}{\mathfrak{h}}^{*} \rightarrow \stackrel{\circ}{\mathfrak{h}^{*}}$ be a linear map defined by $L: \lambda \mapsto \frac{1}{2} \sum_{\alpha \in \stackrel{\circ}{\Delta}} \nu_{\alpha}(\alpha \mid \lambda) \alpha$ where $\nu_{\alpha}$ is $\stackrel{\circ}{W}$-invariant constant. Then $L=a I d_{\mathfrak{h}^{*}}$ where $a \in \mathbb{C}$.

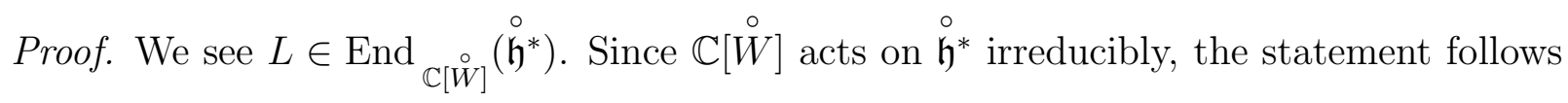
from Schur's lemma.

By this lemma, we see that $-\sum_{\alpha \in \Delta_{t_{-\lambda}}} \mu_{\alpha} \bar{\alpha}=a \lambda$ for some $a \in \mathbb{C}$. The following proposition completes the proof of Theorem 7.5 .

Proposition 7.7. $-\sum_{\alpha \in \Delta_{t_{-\lambda}}} \mu_{\alpha} \bar{\alpha}=h_{\mu}^{\vee} \lambda$. 
Proof. Let $L$ be a linear map defined in the right hand side of (7.16). Owing to Lemma 7.6, we can evaluate the factor $a$ at any element of $\mathfrak{h}^{*}$. Recall that every root system has a quasi-minuscule weight $\nu\left(\theta^{\vee}\right)$, whose properties we have already investigated.

- $A$ is not of type $A_{2 l}^{(2)}$

$$
L\left(\nu\left(\theta^{\vee}\right)\right)=\sum_{\substack{\alpha \in \Delta_{+} \\ \gamma_{\alpha}}} \frac{1}{\alpha}\left\langle\alpha, \theta^{\vee}\right\rangle \alpha=\sum_{\substack{\alpha \in \Delta_{+} \\\left\langle\alpha, \theta^{\vee}\right\rangle \neq 0}} \mu_{\alpha} \alpha+\mu_{\theta} \theta=a \nu\left(\theta^{\vee}\right),
$$

where we have used Lemma 6.2. By applying $(. \mid \theta)$ in the last equality, we obtain

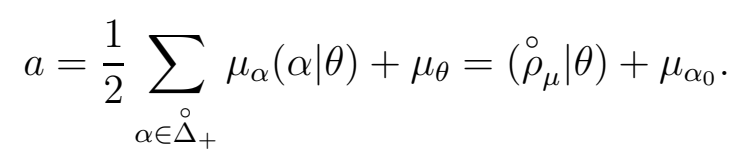

- $A$ is of type $A_{2 l}^{(2)}$

In a similar manner, we have

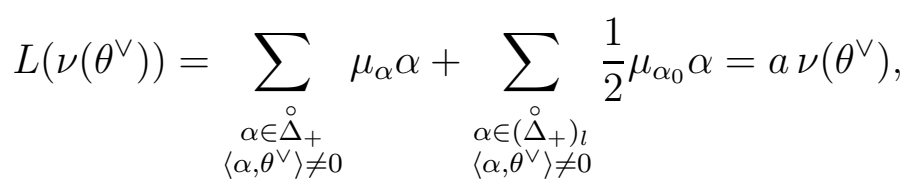

and consequently

$$
a=\left(\stackrel{\circ}{\rho}_{\mu} \mid \theta\right)+\frac{1}{4} \mu_{\alpha_{0}}\left(\rho_{l} \mid \theta\right)=\left(\stackrel{\circ}{\rho}_{\mu} \mid \theta\right)+\mu_{\alpha_{0}},
$$

where $\rho_{l}=\sum_{\alpha \in\left(\grave{\triangle}_{+}\right)_{l}} \alpha=2 \overline{\Lambda_{l}}$.

In any case, $L\left(\nu\left(\theta^{\vee}\right)\right)=h_{\mu}^{\vee} \nu\left(\theta^{\vee}\right)$ and we have $-\sum_{\alpha \in \Delta_{t_{-\lambda}}} \mu_{\alpha} \bar{\alpha}=L(\lambda)=h_{\mu}^{\vee} \lambda$, as required.

Note that we also showed that

$$
\sum_{\alpha \in \grave{\Delta}}(\lambda \mid \alpha)(\mu \mid \alpha)=2 h^{\vee}(\lambda \mid \mu), \quad \text { for } \lambda, \mu \in \stackrel{\circ}{h}^{*},
$$

in the nontwisted root systems. See Corollary 8.7 of [16]. 


\section{Concluding Remarks}

We constructed mutually commuting difference operators by means of the root algebras. Since the operator is represented in a single product of affine $R$-matrices, we had only to pursue the image of each $R$-matrix and therefore suceeded in proving that they act on the characters of the irreducible representations of the affine Lie algebras. However, the procedure of the diagonalization has yet to be solved. Prior to this difficult problem, we may need to show the selfadjointness on the space of the characters with respect to some

inner product, since there is no certainty that they can be diagonalized. In $A_{2}^{(1)}$ case, the selfadjointness was established in [31] for arbitrary level of positive integer.

Since this operator was originally introduced as a quantum many-body system, the selfadjointness should be also an important problem in this sense. In the trigonometric case, we readily see that the Macdonald operators are essentially selfadjoint on the polynomials of exponential since the operators are diagonalized in terms of the Macdonald polynomials. In the elliptic case, however, this problem is less investigated. See, for example, [28, 29] where the two-body system is extensively studied by constructing the explicit eigenvectors, or [17 where the extensibility to positive selfadjoint operators is shown by introducing a certain measure on a torus. These systems correspond to negative levels in terms of the affine Lie algebras and if we treat positive level cases, the measure includes discrete parts.

We hope the construction developed in this paper shed light on these problems.

\section{Acknowledgment}

The author expresses his sincere gratitude to Prof. Masaki Kashiwara and Prof. Tetsuji Miwa who kindly allowed him to speak about his research. Also thanks are due to Prof. Atsuo Kuniba, Prof. Jun'ichi Shiraishi, Prof. Junji Suzuki, Prof. Yuji Yamada, Dr. T. H. Baker, Dr. Goro Hatayama, Prof. Koji Hasegawa, Dr. Takeshi Ikeda, Dr. Tetsuya Kikuchi, Dr. Kazuhiro Hikami and Dr. Akinori Nishino for fruitful discussions and helpful comments. He would like to thank Prof. Miki Wadati for kind interests in this work. He is a 
Research Fellow of the Japan Society for the Promotion of Science.

\section{A Fundamental Functions and Identities}

We define an action of $n=(v, \lambda, u) \in \stackrel{\circ}{\mathfrak{h}} \times \stackrel{\circ}{\mathfrak{h}}^{*} \times \mathbb{C}$ on a holomorphic function $F$ on $Y$ by

$$
(n F)(h):=F\left(t_{-\lambda}(h)-2 \pi i v-(u+\pi i\langle\lambda, v\rangle) K\right) .
$$

Definition A.1. The Heisenberg group is $N_{\mathbb{Z}}=\left\{(v, \lambda, u) \in \stackrel{\circ}{\mathfrak{h}} \times \stackrel{\circ}{\mathfrak{h}}^{*} \times i \mathbb{R} \mid v \in \stackrel{\circ}{Q^{\vee}}, \lambda \in\right.$ $M, u+\pi i\langle\lambda, v\rangle \in 2 \pi i \mathbb{Z}\}$ with multiplication:

$$
(v, \lambda, u)\left(v^{\prime}, \lambda^{\prime}, u^{\prime}\right):=\left(v+v^{\prime}, \lambda+\lambda^{\prime}, u+u^{\prime}+\pi i\left(\left\langle\lambda^{\prime}, v\right\rangle-\left\langle\lambda, v^{\prime}\right\rangle\right)\right) .
$$

Definition A.2. Fix a nonnegative integer $k$. A theta function of level $k$ is a holomorphic function $F$ on the domain $Y$ such that the following two conditions hold:

$$
\begin{aligned}
& n(F)=F \quad \text { for all } n \in N_{\mathbb{Z}} ; \\
& n(F)=e^{-k a} F \quad \text { for all } n=(0,0, a) \in(0,0, \mathbb{C}) .
\end{aligned}
$$

Let $\widetilde{T h^{k}}$ denote the vector space over $\mathbb{C}$ of the theta functions of level $k$. It is known that $\mathscr{O}:=\widetilde{T h^{0}}$ is the set of holomorphic functions of $\langle\delta, h\rangle$.

For $\lambda \in \mathfrak{h}^{*}$ such that level $(\lambda)=k>0$, we set

$$
\Theta_{\lambda}:=e^{-\frac{|\lambda|^{2}}{2 k} \delta} \sum_{t \in T_{M}} e^{t(\lambda)}
$$

It is known that $\left\{\Theta_{\lambda} \mid \operatorname{level}(\lambda)=k\right\}$ is an $\mathscr{O}$-basis of $\widetilde{T h^{k}}$.

Consider the root system of type $A_{1}^{(1)}$. Then $\Pi=\left\{\alpha_{0}, \alpha_{1}\right\} ; M=\mathbb{Z} \alpha_{1} ;\left(\alpha_{1} \mid \alpha_{1}\right)=2$. We have 4 theta functions of level 2 for $k \in \mathbb{Z} / 4 \mathbb{Z}$;

$$
\Theta_{2 \Lambda_{0}+k \overline{\Lambda_{1}}}=e\left(2 \Lambda_{0}\right) \sum_{n \in \mathbb{Z}} e\left(-\frac{1}{2}\left(2 n+\frac{k}{2}\right)^{2} \delta+\left(2 n+\frac{k}{2}\right) \alpha_{1}\right)
$$


where $e(\lambda)(h):=\exp (\langle\lambda, h\rangle)$ for $\lambda \in \mathfrak{h}^{*}$. We see that level $(\rho)=h^{\vee}=2$.

$$
A_{\rho}=\sum_{\substack{\circ \\ w \in W}} \varepsilon(w) \Theta_{w(\rho)}=\Theta_{2 \Lambda_{0}+\frac{1}{2} \alpha_{1}}-\Theta_{2 \Lambda_{0}-\frac{1}{2} \alpha_{1}}=e\left(\rho-\frac{1}{8} \delta\right) \prod_{\alpha \in \Delta_{+}}(1-e(-\alpha)) .
$$

Motivated by these equations, we define theta functions for $\lambda \in \mathfrak{h}^{*}$ and $\gamma>0$ by

$$
\begin{aligned}
\vartheta^{1}(\lambda ; \gamma) & :=\sum_{n \in \mathbb{Z}}(-1)^{n} e\left(-\frac{1}{2}\left(n+\frac{1}{2}\right)^{2} \gamma \delta+\left(n+\frac{1}{2}\right) \lambda\right), \\
\vartheta^{2}(\lambda ; \gamma) & :=\sum_{n \in \mathbb{Z}} e\left(-\frac{1}{2}\left(n+\frac{1}{2}\right)^{2} \gamma \delta+\left(n+\frac{1}{2}\right) \lambda\right), \\
\vartheta^{3}(\lambda ; \gamma) & :=\sum_{n \in \mathbb{Z}} e\left(-\frac{1}{2} n^{2} \gamma \delta+n \lambda\right), \\
\vartheta^{0}(\lambda ; \gamma) & :=\sum_{n \in \mathbb{Z}}(-1)^{n} e\left(-\frac{1}{2} n^{2} \gamma \delta+n \lambda\right),
\end{aligned}
$$

and eta function

$$
\eta(\delta):=e\left(-\frac{1}{24} \delta\right) \prod_{n \in \mathbb{Z}_{\geq 1}}(1-e(-n \delta)) .
$$

Then we have by (A.6) and (A.7)

$$
\vartheta^{1}(\lambda ; \gamma)=e\left(\frac{\lambda}{2}-\frac{1}{8} \gamma \delta\right)(1-e(-\lambda)) \prod_{\substack{\lambda^{\prime} \in\{-\lambda, 0, \lambda\} \\ n \in \mathbb{Z} \geq 1}}\left(1-e\left(-\lambda^{\prime}-n \gamma \delta\right)\right)
$$

It is well known that

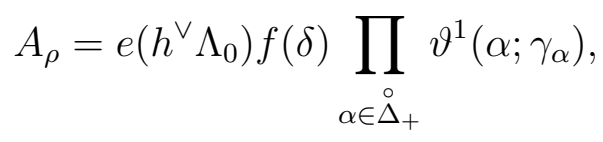

for some function $f(\delta)$ which depends only on $\delta$. We symbolically set

$$
\vartheta^{1 \prime}(0 ; \gamma):=(\eta(\gamma \delta))^{3}
$$

For $\lambda \in \mathfrak{h}^{*}$ and $\nu \in \mathbb{C}$, we define

$$
\sigma_{\nu}(\lambda ; \gamma):=\frac{\vartheta^{1}(\lambda-\nu \delta ; \gamma) \vartheta^{1 \prime}(0 ; \gamma)}{\vartheta^{1}(\lambda ; \gamma) \vartheta^{1}(-\nu \delta ; \gamma)}, \quad \wp^{0}(\lambda ; \gamma):=\left(\frac{\vartheta^{0}(\lambda ; \gamma) \vartheta^{1 \prime}(0 ; \gamma)}{\vartheta^{1}(\lambda ; \gamma) \vartheta^{0}(0 ; \gamma)}\right)^{2}
$$


We see that these theta functions are related to the classical Jacobi theta functions $\vartheta_{j}(z ; \tau)$, Weierstrass function $\wp(z ; 1, \tau)$ and Dedekind eta function $\eta(\tau)$ as

$$
\begin{aligned}
& \vartheta^{1}(\lambda ; \gamma)(h)=-i \vartheta_{1}(\langle\lambda, \stackrel{\circ}{h}\rangle ; \gamma \tau), \\
& \vartheta^{j}(\lambda ; \gamma)(h)=\vartheta_{j}(\langle\lambda, \stackrel{\circ}{h}\rangle ; \gamma \tau), \\
& \wp^{0}(\lambda ; \gamma)(h)=-\frac{1}{4 \pi^{2}}(\wp(\langle\lambda, \stackrel{\circ}{h}\rangle ; 1, \gamma \tau)-\wp(\gamma \tau / 2 ; 1, \gamma \tau)), \\
& \eta(\delta)(h)=\eta(\gamma \tau),
\end{aligned}
$$

where we have set $h=2 \pi i(\stackrel{\circ}{h}-\tau d+u K), \stackrel{\circ}{h} \in \stackrel{\circ}{\mathfrak{h}}$ and $\tau, u \in \mathbb{C}$.

\section{References}

[1] A. J. Bordner and R. Sasaki, Calogero-Moser models III: Elliptic potentials and twisting, preprint, hep-th/9812232.

[2] N. Bourbaki, Groupes et algèbre de Lie, Hermann, Paris, 1969.

[3] I. Cherednik, Double affine Hecke algebras, Knizhnik-Zamolodchikov equations, and Macdonald's operators, Internat. Math. Res. Notices 9 (1992), 171-180.

[4] _ Q Quantum Knizhnik-Zamolodchikov equations and affine root systems, Commun. Math. Phys. 150 (1992), 109-136.

[5] _ Difference-elliptic operators and root systems, Internat. Math. Res. Notices $\mathbf{1}$ (1995), 43-59.

[6] _ Double affine Hecke algebras and Macdonald's conjectures, Ann. Math 141 (1995), 191-216.

[7] _ Intertwining operators of double affine Hecke algebras, Selecta Math. (1997).

[8] P. I. Etingof, Central elements for quantum affine algebras and affine Macdonald's operators, Meth. Res. Lett. 2 (1995), 611-628. 
[9] G. Felder and V. Pasquier, A simple construction of elliptic R-matrices, Lett. Math. Phys. 32 (1994), 167.

[10] G. Felder and A. Varchenko, Elliptic quantum groups and Ruijsenaars models, preprint, q-alg/9704005.

[11] K. Hasegawa, Ruijsenaars' commuting difference operators as commuting transfer matrices, Commun. Math. Phys. 187 (1997), 289-325.

[12] K. Hasegawa, T. Ikeda, and T. Kikuchi, Commuting difference operators arising from the elliptic $C_{2}^{(1)}$-face model, preprint, math.QA/9810062.

[13] K. Hikami and Y. Komori, Diagonalization of the elliptic Ruijsenaars model. correspondence with the Belavin model, Euro. Phys. J. B5 (1998), 583-588.

[14] _, Diagonalization of the elliptic Ruijsenaars model of type-BC, J. Phys. Soc. Jpn. 67 (1998), 4037-4044.

[15] J. E. Humphreys, Reflection groups and Coxeter groups, Cambridge Univ. Press, Cambridge, 1990.

[16] V. G. Kac, Infinite dimensional Lie algebras, Cambridge, Cambridge, 1990.

[17] Y. Komori, Notes on the elliptic Ruijsenaars operators, Lett. Math. Phys 46 (1998), $147-155$.

[18] _ Functional equations arising from the root algebras, in preparation.

[19] Y. Komori and K. Hikami, Elliptic K-matrix associated with Belavin's symmetric R-matrix, Nucl. Phys. B494 (1997), 687-701.

[20] _ Quantum integrability of the generalized elliptic Ruijsenaars models, J. Phys. A30 (1997), 4341-4364.

[21] _ Affine R-matrix and the generalized elliptic Ruijsenaars models, Lett. Math. Phys 43 (1998), 335-346. 
[22] — Conserved operators of the generalized elliptic Ruijsenaars models, J. Math. Phys 39 (1998), 6175-6190.

[23] T. H. Koornwinder, Askey-Wilson polynomials for root systems of type BC, Contemp. Math. 138 (1992), 189-204.

[24] G. Luszig, Affine-Hecke algebras and their graded version, J. Am. Math. Soc. 2 (1989), 599-635.

[25] I. G. Macdonald, Orthogonal polynomials associated with root systems, preprint.

[26] _ Symmetric functions and Hall polynomials, 2nd ed., Oxford Univ. Press, Oxford, 1995.

[27] S. N. M. Ruijsenaars, Complete integrability of relativistic Calogero-Moser systems and elliptic function identities, Commun. Math. Phys. 110 (1987), 191-213.

[28] _ Generalized Lamé functions I. the elliptic case, J. Math. Phys. 40 (1999), $1595-1626$.

[29] _ Generalized Lamé functions II. hyperbolic and trigonometric specializations, J. Math. Phys. 40 (1999), 1627-1663.

[30] Y. Shibukawa and K. Ueno, Completely $\mathbb{Z}$ symmetric $R$ matrix, Lett. Math. Phys. 25 (1992), 239-248.

[31] E. K. Sklyanin, Some algebraic structures connected with the Yang-Baxter equation. representation of quantum algebras., Funk. Anal. i Ego Pril. 17 (1983), 34-48.

[32] J. F. van Diejen, Integrability of difference Calogero-Moser systems, J. Math. Phys. 35 (1994), 2983-2998.

[33] _ Difference Calogero-Moser systems and finite toda chains, J. Math. Phys. 36 (1995), 1299-1323. 